\title{
Advanced OFDM System for Modern Communication Networks
}

\author{
Dr. P R Yadav', Pro. Anil Mishra², Sushil Kumar Chaturvedi ${ }^{3}$ \\ Faculty of Department of Electrical Engineering, JNCT, REWA ${ }^{1,2}$ \\ M.E. Student, Department of Electrical Engineering, JNCT, REWA ${ }^{3}$
}

\begin{abstract}
Orthogonal frequency division multiplexing (OFDM) is a form of digital modulation used in a wide array of communications systems. This paper will explain what OFDM is, why it's important, where it's used, and what test instrumentation is required to maintain it. Perhaps we should first explain what is so special about OFDM. Three things stand out. OFDM is spectrally efficient, carrying more data per unit of bandwidth than such services as GSM and WCDMA. Figure 1 shows a comparison of the spectral efficiency of the leading cellular technologies and how they compare to WLAN and WiMAX. Fourth Generation technology, often referred to as the Long Term Evolution of wireless (LTE) and Ultra Mobile Broadband (UMB) for cellular devices, plans to use OFDM or OFDMA. Orthogonal frequency division multiplexing (OFDM) has become one of the most important modulation methods in many fields, such as high-speed communication systems. It is proposed that OFDM technique can offer variable bandwidth, improved protection to shadow and multipath fading and enhanced robustness thanks to the insertion of the guard interval. In this paper, we introduced the principles of OFDM at first, then analyses the Advantage and disadvantages of OFDM system, and finally discussed its application in contemporary high-speed communication systems, especially in wireless mobile networks.
\end{abstract}

Index Terms: OFDM; ISI; GI; IDFT; DFT

\section{INTRODUCTION}

With the development of wireless communication technologies and the emergence of a large number of multimedia services, the speed and reliability of data transmission are expected to be increased more higher. Orthogonal frequency division multiplexing (OFDM) system is proposed that it can offer variable bandwidth, improved protection to shadow and multipath fading and enhanced robustness thanks to the insertion of the guard interval. So from a certain extent, the OFDM system can solve the bandwidth requirements for data user [1]. And OFDM modulation for wireless network technology is also designed to more fully utilize existing bandwidth, and can very well against the frequency selective fading and narrowband interference. Therefore, using OFDM technique to provide high-speed data transmission has become a hot topic in wireless communication field. II. PRINCIPLES OF OFDMOFDM is a multicarrier transmission technique which divides the available spectrum into many sub carriers, where each sub channel is modulated by a low rate data stream. These subcarriers are made orthogonal to one another, that is, independent from each other.

The orthogonality of the sub channels means that each subcarrier has an integer number of cycles over a symbol period. Owing to this, the spectrum of each sub carrier has a null at the centre frequency for the other subcarriers in the system. This results in no interference between the subcarriers, allowing then to be spaced as closed as theoretically possible, i.e. their spectrum can be overlapped, as shown as figure 1. Compared to conventional frequency division multiplexing (FDM) system, the spectrum efficiency is improved greatly in OFDM system [2].

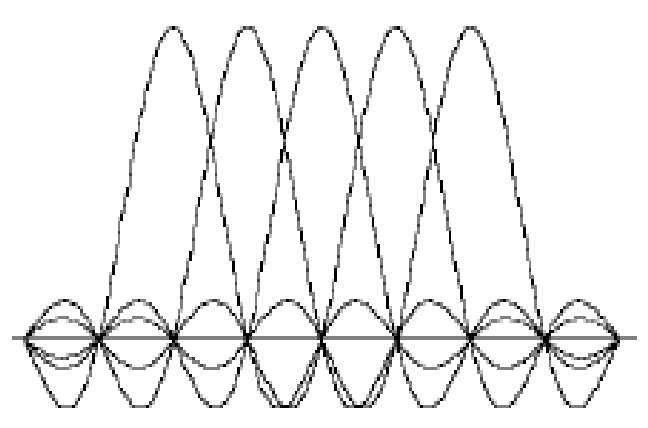

OFDM spectrum

While there are $\mathrm{N}$ sub carries in a OFDM symbol, in which each subcarrier adopts phase shift keying (PSK) or quadrature amplitude modulation (QAM) and carries the data symbol $\operatorname{di}(\mathrm{i}=0,1,2, \ldots \ldots \mathrm{N}-1)$, and $\mathrm{T}$ is the duration of a single OFDM symbol, fi is the frequency of the ith subcarrier, the rectangular function $\operatorname{rect}(\mathrm{t})=1, t \leq T / 2$ ,Then the OFDM symbol s(t), which beginning at $\mathrm{t}=\mathrm{ty}$, can be written phase components after FSK / QAM modulation. Usually the equivalent base-band signal which used to Which, the real and empty parts of $s(t)$ correspond to the same OFDM phase and quadrature values respectively [1]. That is multiplied with the sinusoid components of every sub carriers separately in the actual system, and constitutes the final sub channel signals and the synthesis OFDM symbols. The basic structure of OFDM system is given as Figure 2, in which $\mathrm{fi}=\mathrm{fc}+\mathrm{i} / \mathrm{T}$. The subcarriers demodulation is completed at the receiving end, that is, the same phase and quadrature vector will be transformed to time-domain data to build up the original data and information. 


\section{PRINCIPEL OF OFDM}

In communication systems, the analog implementation of OFDM can be extended to the digital domain by using the discrete Fourier Transform (DFT) and its counterpart, the inverse discrete Fourier Transform (IDFT). These mathematical operations are widely used for transforming data between the time-domain and frequency-domain. These transforms are interesting from the OFDM perspective because they can be viewed as mapping data onto orthogonal subcarriers. For example, the IDFT is used at the transmitter to modulate the original OFDM data, which is to take infrequency-domain data and convert it to time-domain data. In order to perform that operation, the IDFT correlates the frequency-domain input data with its orthogonal basis functions, which are sinusoids at certain frequencies. This correlation is equivalent to mapping the input data onto the sinusoidal basis functions. And DFT can be used at the receiving end to utilize the demodulation.

In practice, OFDM systems are implemented using a combination of fast Fourier Transform (FFT) and inverse fast Fourier Transform (IFFT) blocks that are mathematically equivalent versions of the DFT and IDFT, respectively, but more efficient to implement. An OFDM system treats the source symbols (e.g., the QPSK or QAM symbols) at the transmitter as though they are in the frequency-domain [3]. These symbols are used as the inputs to an IFFT block that brings the signal into the time-domain.

According to figure 2 , if we order $s(t)=0$ in formula(2), ignore the rectangular function, and take the sampling rate of signal $s(t)$ at $T / N$, where $N$ is the number of subcarriers and $\mathrm{T}$ is the IDFT input symbol period mentioned above, then we can attain that $\mathrm{t}=\mathrm{kT} / \mathrm{N}, \quad(\mathrm{k}=0,1, \ldots, \mathrm{N}-1)$. Thus equation (2) can be condensed as:

$$
\text { ( )0 1/ exp } 210 \leq \leq-\mid
$$

Through the N-point IDFT calculation, frequency-domain data symbol becomes time-domain data symbols sk, and it will be sent into the wireless channel after radio frequency (RF) modulation. Each IDFT output data symbols sk is the summation of all $\mathrm{Nm}$ subcarrier signals, thus, the IDFT block provides a simple way to modulate data onto $\mathrm{N}$ orthogonal subcarriers. The block of $\mathrm{N}$ output samples from the IDFT make up a single OFDM symbol. The length of the OFDM symbol is NT where T is the IDFT input symbol period mentioned above. After some additional processing, the time-domain signal so is transmitted across the channel. At the receiver, a DFT block is used to process the received signal and bring it into the frequency-domain. Ideally, the DFT output will be the original data symbol did that were sent to the IDFT at the transmitter: Since the high-speed serial input data stream is assigned into $\mathrm{N}$ parallel sub channels after a serial/parallel conversion in OFDM system, the symbol rate in each sub carry is greatly reduced, and the impact of multipath is weakened evidently. Therefore, the OFDM system is resistant to multipath delay spread. In order to eliminate the inter-symbol interference (ISI) to the minimum, the guard interval (GI) is introduced between the OFDM symbols [2]. The GI length of the guard interval $\mathrm{Tg}$ should be longer than the largest channel delay spread so that the multipath component of such symbol will not interfere with the next one. The OFDM symbols with .In the figure above, the total length of each symbolism

$$
\mathrm{TS}=\mathrm{Tg}+\mathrm{TDFT},
$$

where TS is the total length of asingle OFDM symbol, Tg is the length of guard interval, TDFT the length of OFDM symbol generated by DFT transformation without guard interval. Then at the receiving end, the sampling start time Tx should meet the following formula:

$$
\max \tau<\mathrm{TX}<\mathrm{Tg}(5)
$$

In formula (5), $\max \tau$ is the maximal multipath timespan of each channel. When the sampling start time Tx satisfies formula (5), the disturbance, caused by previous marks, will only exist in the duration of [ ] max $0, \tau$.

When there are more subcarriers, the duration of OFDM symbols Ts is longer than channel's pulse response length. For this reason, the ISI influence is very small. If the guard interval lasting time $\mathrm{Tg}$ conforms $\operatorname{to} \mathrm{Tg} \geqq \max \tau$,

the shortage of ISI may be overcome completely. At the same time, the number of subcarriers' period which existing in the OFDM delay transcription is integer, the delayed signal will generates inter-channel interference ( ICI ) during the demodulation OFDM structure, which is based on IDFT/DFT and inserted guard interval.

Tx is the duration of OFDM symbol, and It meets the following equation:

\section{$\mathrm{TS}=\mathrm{T} g+\mathrm{TFFT}$}

The separate length of guard interval, namely thenumber of sampling point is:

If the number of subcarriers $\mathrm{N}$ is chosen appropriately, it may make the channel influence flatten. And the insertion of guard interval helps to fortify the orthogonality between subcarriers also. So the influence of ISI and the ICI generated by multipath environments can probably be removed totally in OFDM system [4]. The received signal in frequency domain can be displayed as following:

$$
\mathrm{Rn}=\mathrm{HnSn}+\mathrm{Nn}, \mathrm{n}=0, \ldots, \mathrm{N}-1 \text { (11) }
$$

Among them, $\mathrm{Hn}$ is the complex fading coefficient of the nth subcarrier, Nn represents the additive Gaussianwhite noise of the nth subchannel, and its real and empty parts separate from each other while both following zero mean value Gaussian distribution. The noise variance is:

\section{PROPERTIES OF OFDM}

OFDM is a modulation scheme that has recently gained immense popularity in the design of wireless communication systems. The main advantages of OFDM over other communication techniques are that: Makes efficient use of the spectrum by allowing overlap. By dividing the channel into narrowband flat fading sub channels, OFDM is more resistant to frequency selective fading than single carrier systems are. It solves the problem of inter symbol interference (ISI) Using adequate channel coding and interleaving one can recover symbols 
lost due to the frequency selectivity of the channel. Channel equalization becomes simpler than by using adaptive equalization techniques with single carrier systems. It is possible to use maximum likelihood decoding with reasonable complexity. OFDM is computationally efficient by using FFT techniques to implement the modulation and demodulation functions. Is less sensitive to sample timing offsets than single carrier systems are.

Provides good protection against co channel interference and impulsive parasitic noise. Different from single carrier system, the OFDM output signal at receiving end is the summation of which transferred in a great large number of orthogonal sub channels. Thus, there are also disadvantages for OFDM technology:

The OFDM signal has a noise like amplitude with a very large dynamic range; therefore it requires $\mathrm{RF}$ power amplifiers with a high peak to average power ratio. It is more sensitive to carrier frequency offset and drift than single carrier systems due to leakage of the DFT.

\section{APPLICATIONS IN MODERN COMMUNICATION NETWORKS}

OFDM is a transmission technique used to achieve very high data rates. It is applied in several contemporary communication systems, including asynchronous digital subscriber line (ADSL), digital audio/video broadcast systems, wireless area network (WLAN), broadband wireless access (BWA), as well as 3G CDMA or $4 \mathrm{G}$ cellular mobile networks.

In 1997, OFDM was first brought into operation in digital audio broadcast (DAB) standards, which raised by 478 European telecommunications standards institute (ETSI). Nowadays, it has been popularly used as the modulation process in radio broadcasting systems, such as digital audio broadcasting (DAB), digital video broadcasting (DVB), as well as high definition television (HDTV). In WLAN field, both the U.S. IEEE 802.11a and European ETSI Hiperlan/2 standards utilize OFDM technology [5]. IEEE802.11a works in $5 \mathrm{GHz}$ frequency region and employs OFDM modulation in its physical layer. European ETSI Hiperlan/2 standards utilizes both OFDM and link auto adaptation technologies in physical layer, and adopts connection-oriented TDMA/TDD and asynchronous transfer mode (ATM) methods for media access control (MAC) Layer. The maximum data rates can achieve 54Mbit/s. OFDM works well in home and office environments for handling wall reflections and movement within the structure.

OFDM is also suitable for BWA because of its super properties. IEEE 802.16 work group is responsible for the technological works of BWA. It has already developed a new standard for BWA - IEEE 802.16a,which works at $2 \mathrm{GHz}-11 \mathrm{GHz}$ and adopts OFDM technique at physical layer. As a new wireless access technology, IEEE 802.16a may also promote the future development of cellular mobile communication networks. At present, the 3rd generation $(3 \mathrm{G})$ cellular mobile networks based on code division multiple access (CDMA) have been built up and put into service. But the maximum data transmission rate provided by $3 \mathrm{G}$ is only $2 \mathrm{Mbps}$, and is lower in practical system. So $3 G$ network do not suit the demands of data user for multimedia services. In order to promote the $3 \mathrm{G}$ properties, OFDM is introduced into CDMA systems, and the combination of CDMA and OFDM is being investigated by many researchers in mobile field. Nowadays, frequency has already be scarce more and more as the resources of mobile communication systems because of the enlargement of network scale, the increscent of user's requirement for service quantities, as well as the high data transmission rates. So in the $4^{\text {th }}$ generation mobile cellular network (4G), OFDM will get more extensive application because of its fine performance.

\section{CONCLUSIONS}

The multimedia services become one of the developing directions in the wireless communication systems. The multimedia service demands high-speed data transmission rate. Therefore, those techniques which supporting highspeed data transmission is the inevitable development trend in wireless communication networks. OFDM is such a technology that can offer high-efficient and reliable data transmission that has caused much more attentions in modern communication region. With the scale growth of communication networks and the user's demand for atomization, broadband, individualization and mobility, the OFDM technique will be widely used in both cable communication networks and wireless systems.

\section{REFERENCES}

[1] Bingham, J. A. C., Multicarrier Modulation for DataTransmission: An idea whose time has come, IEEECommunications Magazine, Vol.28,no.5, pp.5-14, May1990.J. Clerk Maxwell, A Treatise on Electricity andMagnetism, 3rd ed., vol. 2. Oxford: Clarendon, 1892,pp.68-73

[2] Alen, T. C. H., A. S. Madhukumar, et al. (2003), “Capacity enhancement of a multi-user OFDM systemusing dynamic frequency allocation." Broadcasting,IEEE Transactions on 49(4): pp: 344-353.

[3] Rhee, W. and J. M. Cioffi. Increase in capacity ofmultiuser OFDM system using dynamic subchannelallocation, in Proceedings of Vehicular TechnologyConference Proceedings, 2000, VTC 2000Spring Tokyo,2000 IEEE 51st, 2000. 2: pp: 1085-1089 vol.1082.

[4] J. Stott, The Effects of Phase Noise in COFDM, EBUTechnical Review, Summer 1998.

[5] V. Erceg et al., Channel models for fixed wirelessapplications IEEE 802.16 Broadband Wireless WorkingGroup, IEEE 802.16a-03/01, Tech. Rep., 2003

[6] ETSI DVB-T, "EN 300744 v1.5.1 Digital VideoBroadcasting (DVB) Framing Structure, Channel Codingand Modulation for Digital Terrestrial Television," Tech.Rep., June 2004.

[7] H. Sampath, S. Talwar, J. Tellado,V. Erceg, and A.Paulraj, "A fourthgeneration MIMO-OFDM broadbandwireless system:Design, performance and field trialresults," IEEE.Commun. Mag, vol.40,no.9,pp.143-149,Sep. 2002. 